\title{
Symptomatic Solid Ectopic Cervical Thymus in a 2-Month-Old Infant: Case Report
}

\author{
Case Report () Nursultan Abakir ${ }^{1}$, (1) Fakih Cihat Eravc1 ${ }^{1}$, (1) Ganime Dilek Emlik² \\ ${ }^{1}$ Department of Otorhinolaryngology, Necmettin Erbakan University Meram Faculty of Medicine, Konya, Turkey \\ ${ }^{2}$ Department of Radiology, Necmettin Erbakan University Meram Faculty of Medicine, Konya, Turkey
}

\begin{abstract}
Ectopic cervical thymus (ECT) is a rare pediatric pathology usually with an asymptomatic course.
In fewer cases, the patient may present with dyspnea, hoarseness, stridor, dysphagia and pain. The
mass may present in cystic or solid forms. Solid forms are rare, constituting only 10\% of all ECT
cases and these are mostly symptomatic. We aimed to present the case of an infant with ECT in
the submandibular region that led to shortness of breath. ECTs in the submandibular region are
mostly solid and larger in size, and therefore, likely to result in compressive symptoms. In this
case report, we tried to cover the important aspects of the diagnosis of ECT, its treatment, and
discussed the results with a literature review. We additionally aimed to emphasize the importance
of considering pediatric ECT in the differential diagnosis of pediatric neck mass as well as
considering surgical excision to prevent serious consequences.
Keywords: Thymus, ectopic tissue, neck, tumor, pediatric, case report
\end{abstract}

\section{ORCID ID of the authors:}

N.A. 0000-0002-1752-1289;

F.C.E. 0000-0001-9092-7923;

G.D.E. 0000-0002-7600-0651

Cite this article as: Abakir N, Eravc FC, Emlik GD. Symptomatic Solid Ectopic Cervical Thymus in a 2-Month-Old Infant: Case Report. TurkArch Otorhinolaryngol2021; 59(2): 154-7.

Corresponding Author: Nursultan Abakir; nursultanabakir@gmail.com

Received Date: 21.02.2021 Accepted Date: 31.03 .2021

Content of this journal is licensed under a Creative Commons Attribution 4.0 International License. Available online at www.turkarchotolaryngol.net

\section{(c) (1) ()}

DOl: 10.4274/tao.2021.2021-2-16

\section{Introduction}

The thymic primordium originates from the third pharyngeal pouch during embryogenesis. It disassembles from the pharynx and migrates to the mediastinum, which is its final position in the body (1). During migration, the thymus tissue can be trapped in different locations in the neck region and results in an ectopic cervical thymus (ECT).

ECT is a rare condition and is generally asymptomatic. In some cases, however,
ECT may present with symptoms of mass compression and results in asphyxia or death (2). Additionally, in rare cases, Horner's syndrome can occur (3). Symptomatic ECT patients are usually in the pediatric age group, and it is difficult to differentiate from other pediatric neck masses. Radiological imaging studies can be of tremendous help in distinguishing the condition from other neck mass pathologies.

In this case report, we share a pediatric patient with ectopic thymus who 
presented with shortness in breath. The histopathologic evaluation revealed the solid variant. The aim of this report is to share a rare pediatric neck mass, discuss imaging findings that are useful in differential diagnosis and the management of this pathology.

\section{Case Presentation}

A 2-month-old male baby was admitted to our clinic with swelling in the right submandibular region and a history of shortness of breath for one week. On physical examination, the patient was found healthy except the mass. The lesion was $4 \times 4 \mathrm{~cm}$ in size and mobile, not fixed to the surrounding tissues.

Neck doppler ultrasonography (USG) was performed to evaluate the size of the mass and its relation to the adjacent vascular structures. The Doppler USG revealed a wellcircumscribed, homogeneous, solid mass lesion in the right submandibular region pushing the submandibular gland anteriorly and the major vascular structures medially, as seen in Figure 1. The mass was in the same echo with the thymic tissue and showed significant vascularization with no evidence of invasion to the adjacent structures. In contrastenhanced magnetic resonance imaging (MRI) of the neck, a soft tissue intensity lesion of approximately $5 \times 4 \times 3 \mathrm{~cm}$ was observed in the right submandibular region (Figure 2). When the patient was evaluated together with the MRI and USG findings, the senior author, who is an experienced head and neck radiologist considered the mass as an ectopic thymus because it had the same intensity as the thymus tissue found in the superior-anterior mediastinum. The fine needle aspiration biopsy (FNAB) performed on the patient had not contributed to a definite diagnosis.

The mass was totally excised by dissection from the surrounding tissues under general anesthesia, as shown

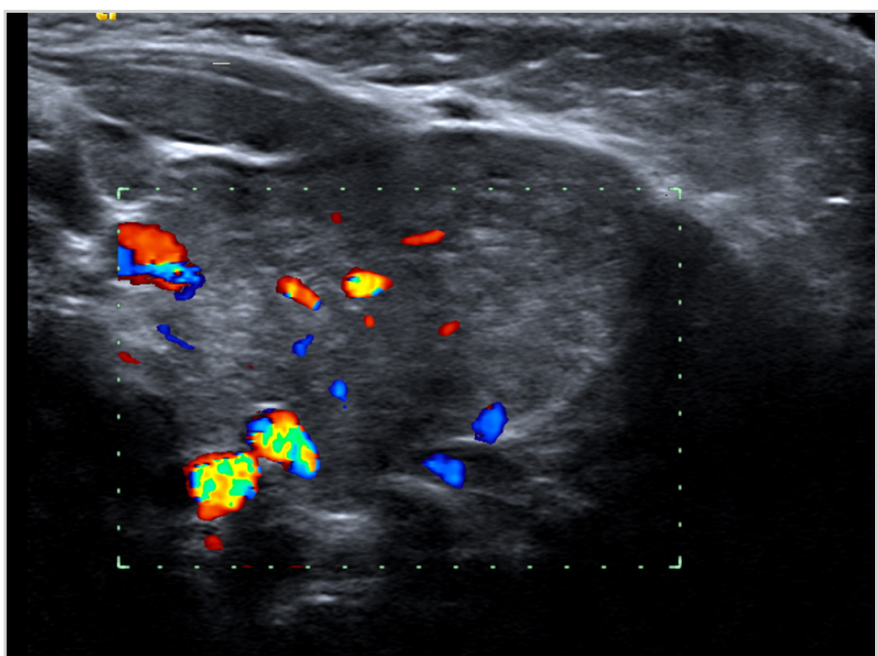

Figure 1. Doppler ultrasonography image of ECT showing the vascularization of the tissue

ECT: Ectopic cervical thymus in Figure 3. The patient did not have any postoperative complications and was discharged.

The diagnosis of the solid form of the ectopic thymus tissue was confirmed in the histopathology report. On the first week of postoperative follow-up, the wound was seen to heal optimally, and the patient's shortness of breath disappeared.

\section{Discussion}

The ectopic thymus can be found in different locations in the neck region as a result of the incomplete descent of the thymic primordia from the $3^{\text {rd }}-4^{\text {th }}$ pharyngeal pouches to the mediastinum. Lesions that remain in the submandibular region during migration are rarely encountered, compared to lesions located in other cervical areas, and tend to be

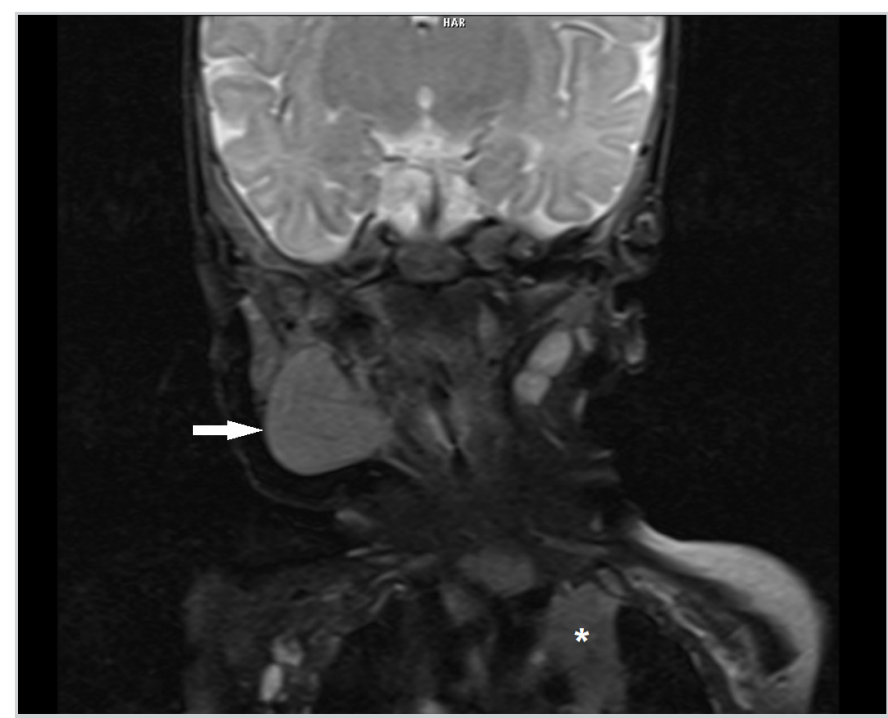

Figure 2. MRI of the neck showing ECT (arrow) is in the same intensity as the normal thymus tissue (asterisk)

MRI: Magnetic resonance imaging ECT: Ectopic cervical thymus

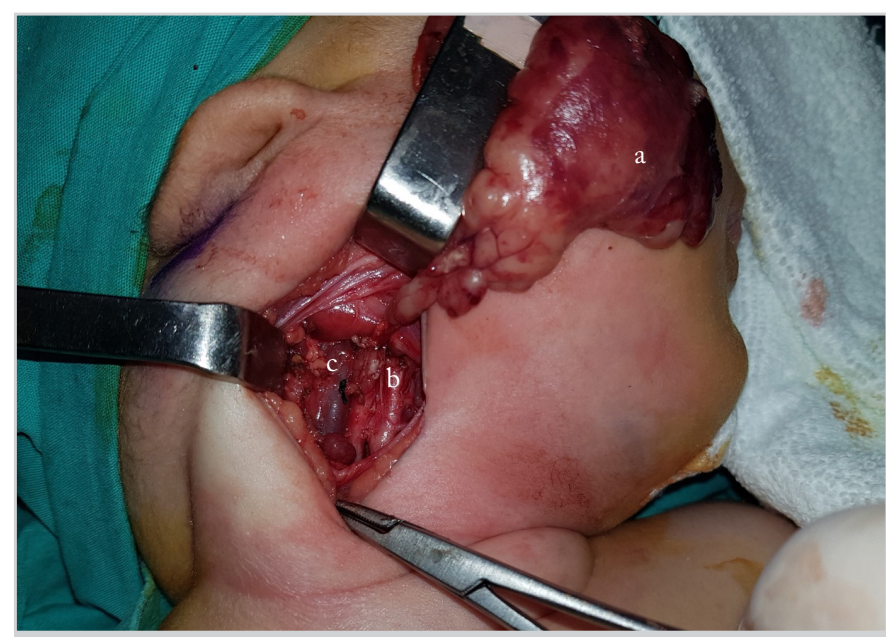

Figure 3. Excision of the solid mass; view highlighting its vicinity to major vasculature. a. solid mass, b. carotid artery, c. internal jugular vein 
larger in size, typically diagnosed at a young age (4). In most cases, ECT is asymptomatic with only $10 \%$ presenting with compressive symptoms including dyspnea, hoarseness, stridor, dysphagia, and pain (5). It is important to differentiate symptomatic ectopic thymus cases from other neck masses to prevent serious consequences.

USG is the most practical and easy method for the diagnosis of ECT. Doppler USG is the most preferred technique for detailed visualization of thymic tissue. In Doppler USG ectopic thymus shows vascular structures corresponding to the echogenic septae with a linear structure (5). As in our patient, USG features revealed blood vessels within the lesion and were more homogenous in structure than the salivary glands.

$\mathrm{MRI}$ is another tool to use in diagnosing soft tissue lesions of the neck. MRI of ECT is homogeneous, has similar features with normal thymus tissue and is hyperintense in T2weighted sequence. Visualization of normal thymic tissue is important before surgical intervention; hence, excision of thymic tissue can lead to immunologic consequences (6). We pre-operatively confirmed the existence of normal thymus in the mediastinum in our patient and identified the similarity of the images of the lesion with normal thymic tissue.

In rare cases, cytopathological examination with FNAB can lead to a certain diagnosis. Some authors refuse to perform FNAB in children because of its controversial diagnostic benefits and general anesthetic requirements $(4,7)$. As the lesion was large, FNAB was performed without general anesthesia in our case. Considering its possible contribution to a differential diagnosis we did perform FNAB. The ectopic thymus can be cystic or solid. Most of the cases are cystic ECT, only 10\% present in solid form (8).

In the recent years, the conservative approach is more accepted in asymptomatic ECT regardless of its location. Purcell et al. (4) proposed a classification based on its location in the neck and claimed that most ectopic thymus are intrathyroidal or in the central neck. In the referred study, only $9 \%$ of the cases were in the submandibular region. Furthermore, lesions located in the submandibular region were larger in size, mostly solid and did not show significant change in size over time (4). If ECT is incidental or there are no symptoms at the time of examination, it is reasonable to follow-up the patient without any surgical intervention.

Although ECT is asymptomatic in most cases, some cases in the literature led to a catastrophic result and patient's death. Ishida et al. (2) reported two infant deaths due to compression of the solid ectopic thymus to the cervical trachea, causing sudden infant death syndrome. Modabber et al. (3) reported a congenital case of Horner's syndrome associated with ECT in a 19-month-old patient. Considering all these symptoms of ECT, even if they are not prevalent, we recommend the total excision of the lesion to avoid an unwanted outcome.

\section{Conclusion}

Although rare, ECT should be considered in the differential diagnosis of mass lesions of the neck region in pediatric patients. In imaging modalities, the normal location of the thymus should also be scanned along with the region of the mass. The surgical treatment should be planned only if the thymus gland is observed in the normal location. Even they are mostly asymptomatic, a solid variant of ECT in the submandibular location should be excised given the risk of airway compression.

Informed Consent: A written informed consent was obtained from the patient's parents.

Peer-review: Externally peer-reviewed.

\section{Authorship Contributions}

Concept: F.C.E., N.A., Design: F.C.E., N.A., Supervision: G.D.E., Materials: F.C.E., N.A., G.D.E., Data Collection and/or Processing: N.A., G.D.E., Analysis and/or Interpretation: F.C.E., G.D.E., Literature Search: N.A., Writing: F.C.E., Critical Reviews: F.C.E., G.D.E.

Conflict of Interest: The authors declare that they have no conflict of interest to disclose.

Financial Disclosure: The authors declare that this study has not received any financial support.

\section{References}

1. Gordon J, Manley NR. Mechanisms of thymus organogenesis and morphogenesis. Development 2011; 138: 3865-78. [Crossref]

2. Ishida T, Kotani H, Miyao M, Abiru H, Kawai C, Osamura T, et al. Ectopic cervical thymus associated with infant death: 2 case reports and literature review. Int J Pediatr Otorhinolaryngol 2013; 77: 1609-12. [Crossref]

3. Modabber M, Saint-Martin C, Khan A, Zakrzewski H, Toffoli D. Infantile Horner syndrome due to ectopic cervical thymus gland. Can J Ophthalmol 2019; 54: e10-2. [Crossref]

4. Purcell PL, Marquez Garcia J, Zawawi F, Propst EJ, Papsin BC, Blaser SI, et al. Ectopic cervical thymus in children: clinical and radiographic features. Laryngoscope 2020; 130: 1577-82. [Crossref]

5. Cacciaguerra S, Rizzo L, Tranchina M, Cutrona D, Di Benedetto A. Ultrasound features of ectopic cervical thymus in a child. Pediatr Surg Int 1998; 13: 597-9. [Crossref] 
6. Wells WJ, Parkman R, Smogorzewska E, Barr M. Neonatal thymectomy: does it affect immune function? J Thorac Cardiovasc Surg 1998; 115: 1041-6. [Crossref]

7. Zhaoqiang Z, Qingbin Z, Lei C, Dan C, Yu C, Wangxiang Y, et al. Infant ectopic cervical thymus one case report: diagnostic and management difficulties. J Craniomaxillofac Surg 2012; 40: 701-5. [Crossref]

8. Prasad TR, Chui CH, Ong CL, Meenakshi A. Cervical ectopic thymus in an infant. Singapore Med J 2006; 47: 68-70. [Crossref] 